\title{
openheart Coronary artery ectasia: prevalence, angiographic characteristics and clinical outcome
}

\author{
Nadav Asher Willner (D), Scott Ehrenberg, Anees Musallam, Ariel Roguin
}

To cite: Willner NA, Ehrenberg S, Musallam A, et al. Coronary artery ectasia: prevalence, angiographic characteristics and clinical outcome. Open Heart 2020;7:e001096. doi:10.1136/ openhrt-2019-001096

Received 27 May 2019 Revised 20 November 2019 Accepted 27 January 2020
Check for updates

\section{(C) Author(s) (or their} employer(s)) 2020. Re-use permitted under CC BY-NC. No commercial re-use. See rights and permissions. Published by BMJ.

Department of Cardiology, Rambam Health Care Campus, Haifa, Israel

\section{Correspondence to}

Dr Nadav Asher Willner; nadav. willner@gmail.com

\begin{abstract}
Objective Determine coronary artery ectasia (CAE) prevalence and clinical outcome in a large cohort of patients underwent coronary angiography.

Methods In an 11-year period, between 2006 and 2017, 20455 coronary angiography studies were performed at a large university centre. Patients diagnosed with CAE based on procedure report were included in the final analysis. Results CAE was diagnosed in 174 out of 20455 studies ( $0.85 \%$ per total angiograms, 161 patients). Patients' average age was $59.6 \pm 11.2$ years old with male predominance $(90.7 \%)$. Diffuse ectasia morphology was most common $(78.9 \%)$, followed by fusiform $(16.1 \%)$ and saccular (5\%). Mixed CAE and atherosclerotic heart disease (ASHD) was present in $75.2 \%$ of the patients and isolated CAE in $24.8 \%$. The most common coronary artery involved was the right coronary artery (RCA) $(79 \%)$. Following index angiography, all the isolated CAE group was managed conservatively, while $67 \%$ of the mixed CAE-ASHD group underwent coronary intervention. In an average follow-up of $6 \pm 3.6$ years, adverse clinical event (a composite endpoint of any death, cerebrovascular accident, myocardial infarction, thromboembolic event, bleeding and stent thrombosis) occurred in $48.8 \%$ of the mixed CAE-ASHD group compared with $25 \%$ in the isolated CAE group $(p<0.05)$.

Conclusions CAE is a rare phenomenon. The most common artery involved was the RCA, and the diffused type of CAE was the most frequent. Most patients with CAE have also concomitant ASHD, and those patients have higher mortality and complications rate, compared with isolated CAE disease.
\end{abstract}

\section{INTRODUCTION}

Coronary artery ectasia (CAE), the aneurysmal dilatation of a coronary artery, is defined as segmental dilatation with a diameter of 1.5 times compared with an adjacent normal coronary artery. ${ }^{1} \mathrm{CAE}$ is relatively uncommon with a prevalence of $1.2 \%-4.9 \%$ according to previous studies. ${ }^{2}$ Classification is usually based on shape and extent of involvement of the coronary arteries: an arterial aneurysm is usually reserved for focal dilatation, whereas the term ectasia is used to describe an elongated and extended widening of a tubular structure. ${ }^{3}$

\section{Key questions}

What is already known about this subject?

- Coronary artery ectasia (CAE) is relatively uncommon finding in coronary angiography. Risk factors for CAE and classical atherosclerotic disease partially overlap, but isolated CAE also exists. The right coronary artery is usually involved.

What does this study add?

- This study adds a reliable estimation of CAE incidence and clinical outcomes based on a very large population size and a long follow-up period.

How might this impact on clinical practice?

- This study results should encourage closer clinical follow-up on patients with CAE, especially those having a concomitant atherosclerotic coronary disease. At the same time, it may discourage the use of anticoagulation treatments for patients with isolated CAE.

\section{Strengths and limitations of this study}

- Population size of present study (161 patients) is larger than most previous studies.

- Long-term clinical follow-up period of 10 years.

- Systematic evaluation of coronary artery ectasia (CAE) angiograms by an experienced invasive cardiologist.

- Possible underestimation of the actual incidence of CAE due to non-reported or recognised cases.

- No healthy controls compared with patients with CAE.

Risk factors for CAE and classical atherosclerotic disease partially overlap. Most patients with CAE have atherosclerotic heart disease (ASHD) concomitantly. This raises the questions of possible common pathophysiology or maybe CAE being a variant of ASHD. Nevertheless, CAE can also be isolated, hence with no atherosclerotic stenotic arteries, a situation conceivably prone to turbulent flow with elevated ischaemic risk. ${ }^{45}$

The clinical relevance in general, and the appropriate medical management of $\mathrm{CAE}$ 
specifically, is ill defined, as no randomised prospective studies exists. Most studies showed similar cardiovascular risk for patients with concomitant CAE and ASHD compared with ASHD only, while isolated CAE has a good prognosis and a low risk for ischaemic events. ${ }^{6} \mathrm{CAE}$ patients with acute myocardial infarction (MI) undergoing percutaneous coronary intervention (PCI) appear to have more complicated procedures with frequent adverse events. $^{7}$

We conducted a retrospective analysis aimed at identifying the prevalence of CAE, inspected disease extent and morphology, and finally, investigated clinical characteristics and outcomes of this unique condition.

\section{METHODS}

In an 11-year period, between the years 2006 and 2017, a total of 20455 coronary angiography procedures were performed at a 1000-bed university centre. Out of these, 174 reports included the term 'coronary ectasia' or 'ectatic coronary disease'. Angiograms were reviewed by the authors for final confirmation of the diagnosis of CAE. The study database included clinical and angiographic details obtained from angiography reports.

CAE morphology was defined as follows ${ }^{2}$ : (1) diffused: extended disease involving long segments of the artery; (2) fusiform: transverse diameter of ectasia is less than the longitudinal dimension; and (3) saccular: transverse diameter of ectasia is greater than the longitudinal dimension. When more than one morphology was evident, the more visually prominent one was defined.

\section{Outcomes and definitions}

The primary outcome of interest was patient-oriented composite endpoint (POCE; a composite of any death, cerebrovascular accident (CVA; including transient ischaemic event), any MI (defined according to the European Society of Cardiology fourth universal definition of MI) with and without any coronary revascularisation, any thromboembolic event or bleeding and rate of stent thrombosis). In case of more than one procedure per patient during the above period, only procedures indicated because of the above-mentioned POCE were considered as primary outcome.

\section{Statistical analysis}

Baseline characteristics were reported as mean and SD for continuous variables, and number and percentages for categorical variables. Statistical differences between baseline characteristics were reported using a t-test for continuous variables and $\chi^{2}$ test for categorical variables. For clinical outcomes analysis, time-to-event KaplanMeier models and multivariate Cox proportional hazards models were used, assuming all events are equally weighted (events censored at patient's death or last day of follow-up). SPSS Statistics V.22 was used for statistical analysis.

No informed consent forms were needed (retrospective analysis).

\section{RESULTS}

CAE was diagnosed in 174 out of 20455 catheterisation reports analysed $(0.85 \%$ per total angiograms, 161 patients). Patients' average age was 59.6 \pm 11.2 years old (range 33-87) with male predominance $(\mathrm{n}=146,90.7 \%)$. Ninety-eight patients $(60.9 \%)$ were treated with aspirin before admission, two patients were pretreated with anticoagulation and the rest $(37.8 \%)$ had no previous antiplatelet medications. The indication for pretreatment with antiplatelets or anticoagulants was not documented clearly in most patients' electronic medical records.

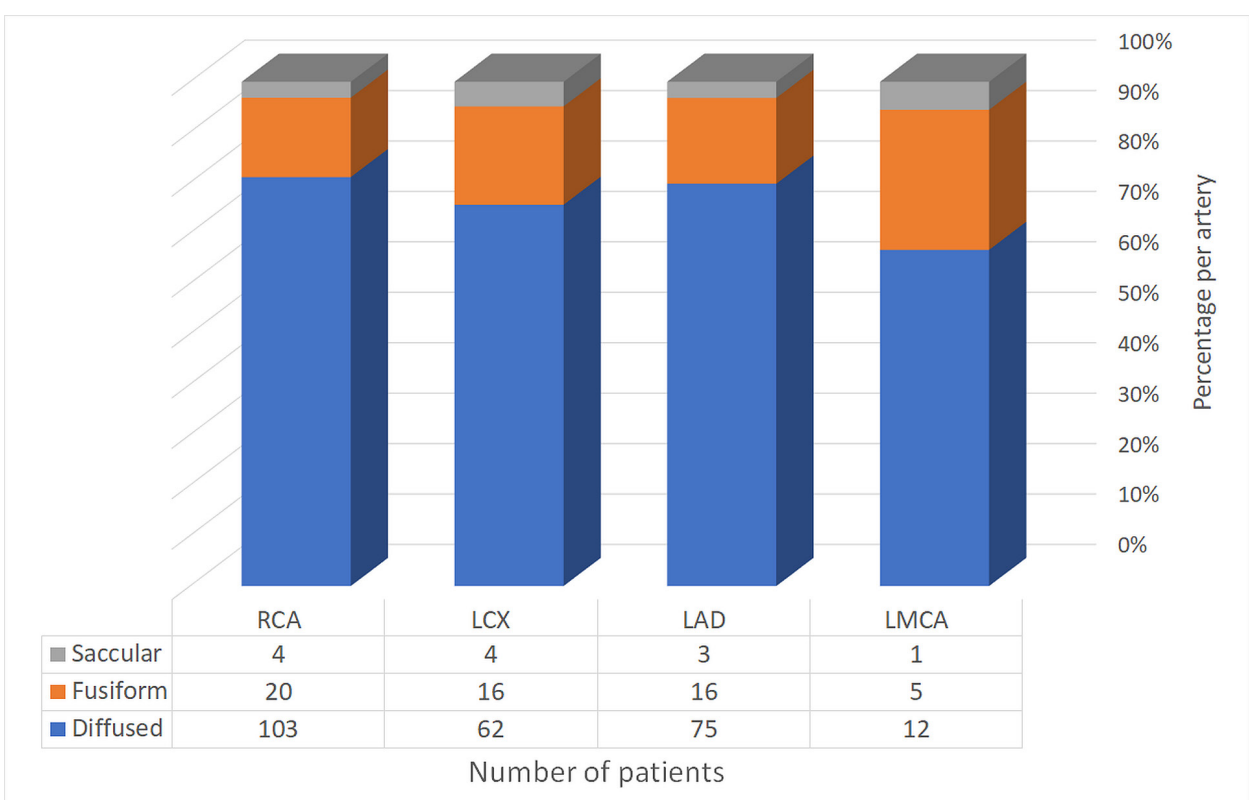

Figure 1 Coronary ectasia anatomical form by coronary artery. LAD, left anterior descending; LCX, left circumflex; LMCA, left main coronary artery; RCA, right coronary artery. 
Table 1 Patients' background and clinical indication for cardiac catheterisation at index event

\begin{tabular}{|c|c|c|c|c|c|}
\hline & & & Isolated CAE & Mixed CAE and ASHD & $P$ value \\
\hline \multirow[t]{5}{*}{ Demographic background } & Age $($ mean $\pm S D)$ & & $57 \pm 12$ & $60 \pm 11$ & 0.08 \\
\hline & \multirow[t]{2}{*}{ Gender (N, \%) } & Female & $5(12.5)$ & $10(8.3)$ & \multirow[t]{2}{*}{0.42} \\
\hline & & Male & 35 (87.5) & $111(91.7)$ & \\
\hline & Smoking (N, \%) & & $24(60)$ & 85 (70.8) & 0.24 \\
\hline & Hypertension (N, \%) & & $30(75)$ & $91(75.8)$ & 1.00 \\
\hline \multirow{9}{*}{$\begin{array}{l}\text { Clinical indication for } \\
\text { coronary angiography } \\
(\mathrm{N}, \%)\end{array}$} & \multirow[t]{3}{*}{$\operatorname{ACS}(N, \%)$} & NSTEMI & $24(60)$ & $55(45.5)$ & \multirow[t]{9}{*}{0.052} \\
\hline & & STEMI & $0(0)$ & $25(20.7)$ & \\
\hline & & UAP & $1(2.5)$ & $4(3.3)$ & \\
\hline & Stable angina pectoris & & $5(12.5)$ & $20(16.5)$ & \\
\hline & Positive stress test & & $6(15)$ & $6(5)$ & \\
\hline & VT & & $0(0)$ & $1(0.8)$ & \\
\hline & LV dysfunction & & $1(2.5)$ & $1(0.8)$ & \\
\hline & Chest pain & & $0(0)$ & $1(0.8)$ & \\
\hline & Other & & $3(7.5)$ & $8(6.6)$ & \\
\hline \multirow{10}{*}{$\begin{array}{l}\text { Angiographic } \\
\text { characteristics }\end{array}$} & \multirow{3}{*}{$\begin{array}{l}\text { Number of involved arteries } \\
(\mathrm{N}, \%)\end{array}$} & 1 & $11(27.5)$ & $43(35.5)$ & \multirow[t]{3}{*}{0.049} \\
\hline & & 2 & $12(30)$ & $47(38.8)$ & \\
\hline & & $\geq 3$ & $17(42.5)$ & $31(25.6)$ & \\
\hline & \multirow[t]{3}{*}{ Ectasia morphology (N, \%) } & Diffused & 37 (92.5) & $90(74.4)$ & \multirow[t]{3}{*}{0.042} \\
\hline & & Fusiform & $3(7.5)$ & $23(19)$ & \\
\hline & & Saccular & $0(0)$ & $8(6.6)$ & \\
\hline & \multirow{4}{*}{$\begin{array}{l}\text { Artery involved } \\
\text { (N, \% of group) }\end{array}$} & LMCA & $3(7.5)$ & $15(12.4)$ & 0.56 \\
\hline & & LAD & $31(77.5)$ & $63(52.2)$ & 0.05 \\
\hline & & LCX & $18(45)$ & $64(52.9)$ & 0.46 \\
\hline & & $\mathrm{RCA}$ & $34(85)$ & $93(76.9)$ & 0.37 \\
\hline Total (N, \% of total) & & & $40(24.8)$ & $121(75.2)$ & \\
\hline
\end{tabular}

ACS, acute coronary syndrome; ASHD, atherosclerotic heart disease; CAE, coronary artery ectasia; LAD, left anterior descending; LCX, left circumflex; LMCA, left main coronary artery; LV, left ventricle; MI, myocardial infarction; NSTEMI, non-ST elevation MI; RCA, right coronary artery; STEMI, ST elevation MI; UAP, unstable angina pectoris; VT, ventricular tachycardia.

The most frequent clinical indication for catheterisation was acute coronary syndrome (ACS) (109 patients, $67.7 \%)$, followed by stable angina pectoris (25 patients, $15.5 \%)$ and positive stress test (12 patients, $7.4 \%)$. Onethird $(33.5 \%)$ of the patients had only one ectatic coronary vessel, 57 (35.4\%) had two and 50 patients $(31.1 \%)$ had three or more vessels involved. The right coronary artery (RCA) was most frequently involved $(78.9 \%$ of total patients), followed by the left anterior descending (LAD; 58.4\%), left circumflex (LCX; 50.9\%), left main coronary artery (LMCA; 11.2\%) and the posterior descending artery $3.2 \%)$. The anatomical morphologies of ectasia were diffused $(78.9 \%)$, fusiform $(16.1 \%)$ and saccular $(5 \%)$, with no differences between specific arteries (figure 1).

Based on index coronary angiography, 40 patients $(24.8 \%)$ were classified as isolated CAE, and 121 patients (75.2\%) were classified as mixed CAE and ASHD. No significant differences in age, gender, smoking, hypertension or clinical indication for coronary angiography were found between the two groups (see table 1). All patients presenting with ST segment elevation MI (STEMI) belonged to the mixed group (but that difference was marginally significant, $\mathrm{p}=0.052$ ).

In the isolated CAE group, $42.5 \%$ had three or more coronary arteries involved compared with only 25.6\% in the mixed group $(\mathrm{p}=0.049)$. In both groups, diffused morphology predominated significantly among the three types of ectasia $(92.5 \%$ and $74.4 \%$, respectively, $\mathrm{p}<0.05)$. Finally, the RCA was most frequently involved in both groups, followed by the LAD in the isolated-CAE group and the LCX in the mixed group (see table 1).

In the isolated CAE group, all patients $(n=40)$ were managed conservatively (ie, without PCI), irrespective of their clinical indication for catheterisation, yet $24.4 \%$ received dual antiplatelets therapy at discharge. In the mixed CAE and ASHD group $(\mathrm{n}=121), 37.2 \%$ were managed conservatively, $67 \%$ underwent PCI, and one patient $(0.8 \%)$ was referred for coronary artery bypass surgery. All patients underwent PCI were discharged with dual antiplatelets therapy. In $60 \%(n=45 / 75)$ of the patients who underwent PCI, the infarct-related artery 


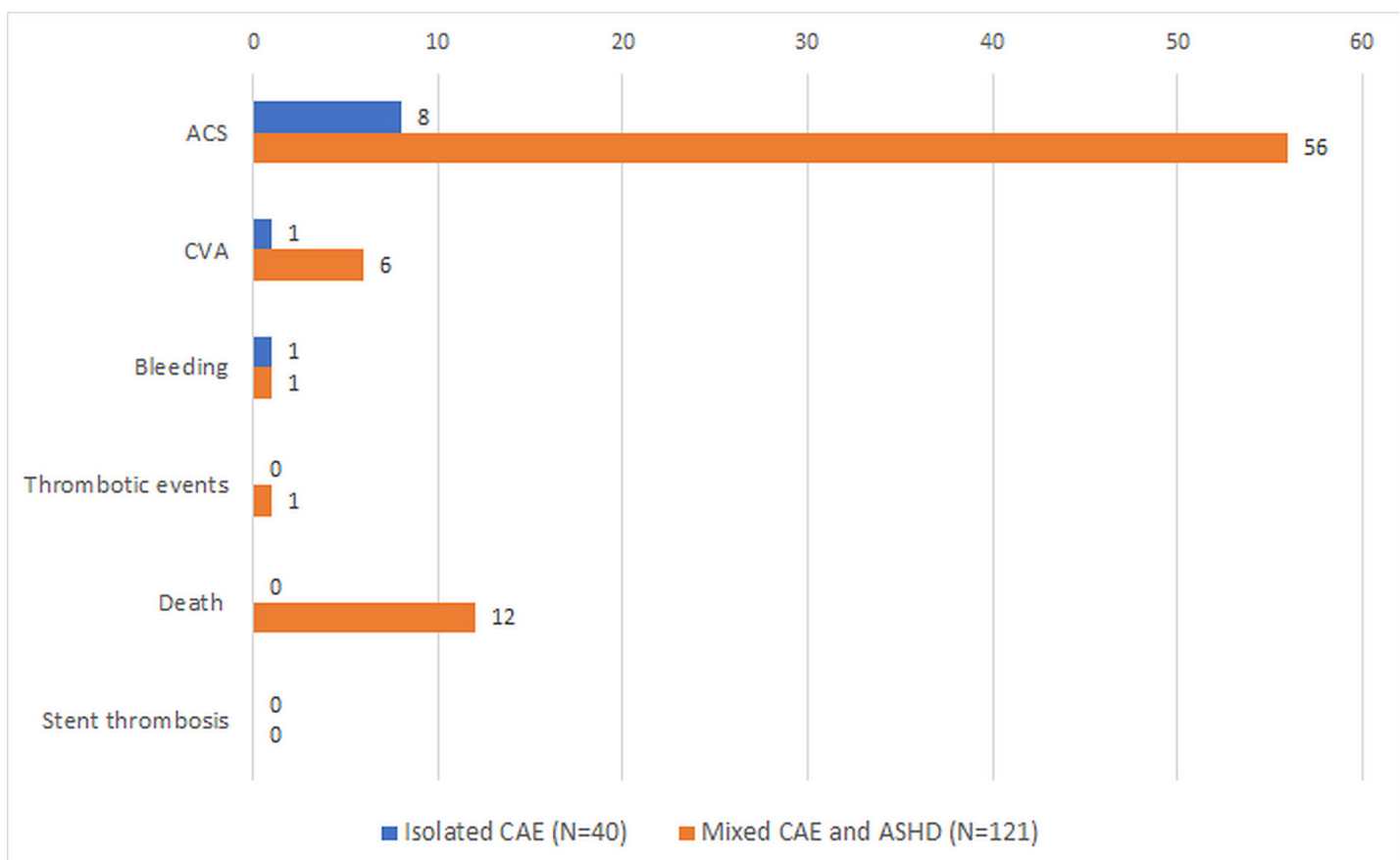

Figure 2 Major adverse cardiovascular events by coronary ectasia disease type. Patients with mixed CAE disease had higher rate of ACS. No patients died in the isolated CAE group during follow-up period. Bleeding included one event of intracranial haemorrhage, and thromboembolic events included two deep vein thrombosis events. ACS, acute coronary syndrome; ASHD, atherosclerotic heart disease; CAE, coronary artery ectasia; CVA, cerebrovascular accident.

that was treated was also ectatic. IIbIIIA inhibitor (ie, tirofiban or eptifibatide) was used during or after coronary angiography in $24(14.9 \%)$ patients.

Average follow-up time was $6 \pm 3.6$ years. Sixty-nine patients (42.2\% of total patients) had POCE (figure 2): 13 patients $(8.1 \%)$ died, 59 events of ACS, 7 events of CVA, 2 events of bleeding-intracranial haemorrhage and one thromboembolic event-deep vein thrombosis

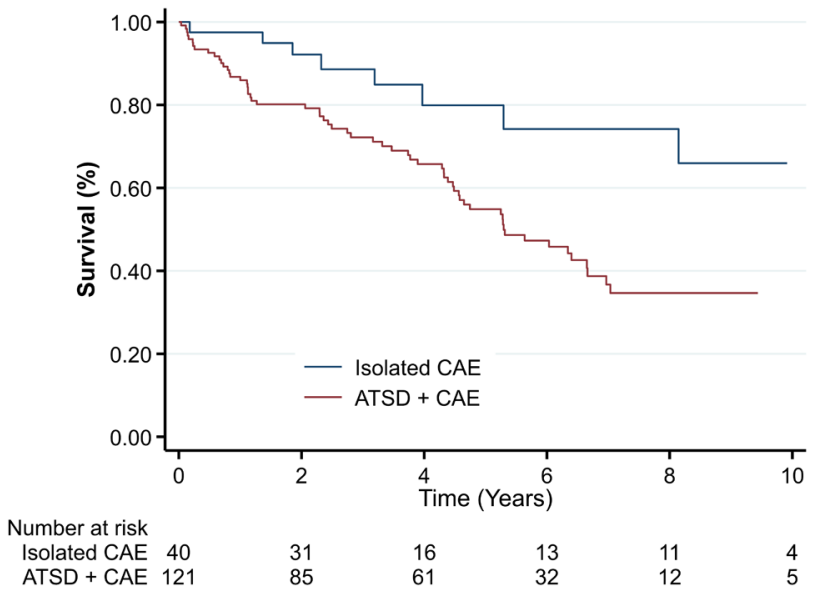

Figure 3 Kaplan-Meier survival curve for ACS-free percentage during follow-up time. POCE prevalence was significantly higher in the mixed CAE and ATSD group compared with the isolated CAE group $(p<0.05)$. ACS, acute coronary syndrome; ATSD, atherosclerotic heart disease; $\mathrm{CAE}$, coronary artery ectasia; POCE, patient-oriented composite endpoint.
$(38.5 \%, 4.3 \%, 1.2 \%$ and $0.6 \%$, respectively). No stent thrombosis events reported. The prevalence of POCE was significantly higher in the mixed CAE and ASHD group, compared with the isolated CAE group $(48.8 \%$ vs $25 \%$, $\mathrm{p}=0.008$, figure 2). Multivariate Cox proportional hazards model found only having mixed CAE and ASHD, and hypertension, to be associated with POCE (mixed disease: HR 2.65 (95\% CI 1.3 to 5.3), $\mathrm{p}=0.006$, and hypertension: HR 2.04 (95\% CI 1.07 to 3.9), p=0.03). Kaplan-Meier curve for POCE comparing mixed versus isolated CAE disease using cumulative incidence is shown in figure 3 .

During follow-up period, 57 of $121(40.4 \%)$ patients in the mixed CAE-ASHD group underwent coronary angiography, with ACS being the clinical indication in most of them $(89.5 \%)$. In $35.1 \%$, the culprit lesion (CL) was ectatic, with $14 \%$ target lesion failure (ie, the CL was identical to CL at index angioplasty). A percentage of $52.6 \mathrm{had}$ subsequent revascularisation (PCI). In the isolated CAE group, only 7 of 40 patients $(17.5 \%)$ with ACS (virtually all had unstable angina) underwent angiography, with no patients having also subsequent PCI.

\section{DISCUSSION}

The size of the population of the present study of 161 patients is larger than most previous studies. ${ }^{2}$ The frequency of reported CAE per total angiograms in our study was $0.85 \%$, and it is lower compared with CAE prevalence in previous studies. Most patients with CAE have also concomitant ASHD, and those patients have higher mortality and complications rate, compared with isolated 
CAE disease. Preadmission antiplatelet treatment did not correlate with clinical outcome or angiographic characteristics. Isolated CAE prevalence was $0.2 \%$, as previously described in medical literature. ${ }^{16}$ Male predominance of more than $90 \%$ resembles prior findings. ${ }^{78}$

Previous studies comparing CAE angiograms with and without concomitant ASHD found similar ratio in these groups' sizes as in our study, with majority of patients (1:4) having concomitant CAE and ASHD. ${ }^{46}$ Patients with isolated CAE had more extended disease in terms of both number of arteries involved $(42.5 \%$ had three ectatic vessels) and dominant diffused morphology (92.5\%).

Lam and $\mathrm{Ho}^{9}$ found that $60 \%$ of mixed CAE and ASHD had only one ectatic vessel and only $20 \%$ had more extended disease; the extent of disease in isolated CAE is not consistently reported in the literature. In agreement with previous publications, the RCA was the most commonly affected coronary artery, followed by the LAD, LCX and LMCA. ${ }^{4910}$ Of note, LMCA was ectatic in a relatively high percent $(11.2 \%)$ of patients. Diffused ectasia morphology dominated in all patients and all arteries.

While the majority of patients presented with angina (ACS and stable disease), only half of them eventually underwent PCI; the remaining half were managed conservatively. Analysing this in the context of isolated versus mixed disease, the discrepancy is even more prominent-virtually all patients presenting with STEMI belonged to the mixed CAE and ASHD group, while all isolated CAE patients were treated only medically. Moreover, in two-thirds of PCI procedures in patients with CAE, the artery that was treated was actually not ectatic. Those findings might reflect different underlying pathophysiological mechanisms between the groups. While the mixed group patients presented as 'classical' ASHD, clinically and angiographically, isolated CAE patients possibly suffer from ectasia related ischaemia, secondary to slow flow and/or thrombotic events. This latter mechanism in isolated CAE is supported by both clinical trials ${ }^{11}{ }^{12}$ and cardiac imaging. ${ }^{13} 14$

During the follow-up of 6 years in average, mortality rate from all causes was $4.3 \%$ in all patients, all of them coming from the mixed CAE and ASHD group. Different study designs restrict direct comparison with past publications, but Swaye $e t a l^{1}$, similarly reported reduced survival rate only in patients with concomitant $\mathrm{CAE}$ and ASHD. ${ }^{1}$ The significantly higher rates of POCE in the mixed CAE and ASHD group compared with isolated CAE is definitely attributed to ACS (other adverse events were rare). Using multivariate Cox regression model, we found that only hypertension and concomitant ASHD were associated with POCE. Unfortunately, we had no control group of healthy patients or patients with ASHD only. Nevertheless, those findings resembles most, if not all, previous studies that concluded that CAE itself does not contribute to the inherent cardiovascular risk of ASHD, and the risk for MI among isolated CAE patients is very low. ${ }^{7}$ This conclusion is also supported by the extremely low rate of repeated angiography and an even lower rate of revascularisation found in our study, in patients with isolated CAE.

A recent study from Japan showed increased prevalence of death and MI in the CAE group among 1698 patients with acute $\mathrm{MI}^{15}$, concluding that the presence of CAE predicted future cardiac events in patients with acute MI. The authors also suggested patients with CAE might benefit from anticoagulation treatment. Since no data regarding concomitant ASHD and CAE was given by the authors, we find those conclusions with limited value. Anticoagulation treatment for CAE, though recommended by some authors in the past, was never supported in large trials. Our findings demonstrate no increased clinical event risk in isolated CAE compared with mixed CAE and ASHD and may indirectly support the common practice against anticoagulation therapy in isolated CAE patients.

Our study has a few limitations. First, relying on reports and electronic medical records only might cause underestimation of the actual incidence/prevalence of CAE and POCE. Second, we have no healthy controls compared with CAE patients; nevertheless, retrospective study is appropriate given the rare frequency of CAE. Further research using matching of cohorts on sex, age and traditional risk factors would provide stronger evidence of a potential association of CAE in events.

\section{CONCLUSIONS}

In this retrospective study of 20455 consecutive patients who underwent angiography for a variety of clinical cardiac indications, $174(0.85 \%)$ angiograms demonstrated definitive CAE. The majority of cases possessed a mixed CAE and ASHD, while isolated CAE was scarce. The most common coronary artery involved was the RCA, and diffused morphology was exceedingly frequent. During the follow-up period, POCE predominated the mixed CAE-ASHD group, while isolated CAE seem to have more benign prognosis.

Contributors All authors equally contributed with literature review, data analysis and writing of the final manuscript.

Funding The authors have not declared a specific grant for this research from any funding agency in the public, commercial or not-for-profit sectors.

Competing interests None declared.

Patient consent for publication Not required.

Ethics approval The study was approved by the institutional review board (approval number RMB-18-0267).

Provenance and peer review Not commissioned; externally peer reviewed.

Data availability statement Data are available on reasonable request.

Open access This is an open access article distributed in accordance with the Creative Commons Attribution Non Commercial (CC BY-NC 4.0) license, which permits others to distribute, remix, adapt, build upon this work non-commercially, and license their derivative works on different terms, provided the original work is properly cited, appropriate credit is given, any changes made indicated, and the use is non-commercial. See: http://creativecommons.org/licenses/by-nc/4.0/.

\section{ORCID iD}

Nadav Asher Willner http://orcid.org/0000-0003-4750-3425 


\section{REFERENCES}

1 Swaye PS, Fisher LD, Litwin P, et al. Aneurysmal coronary artery disease. Circulation 1983;67:134-8.

2 Devabhaktuni S, Mercedes A, Diep J, et al. Coronary artery Ectasia-A review of current literature. Curr Cardiol Rev 2016;12:318-23.

3 Markis JE, Joffe CD, Cohn PF, et al. Clinical significance of coronary arterial ectasia. Am J Cardiol 1976;37:217-22.

4 Gunes Y, Boztosun B, Yildiz A, et al. Clinical profile and outcome of coronary artery ectasia. Heart 2006;92:1159-60.

5 Brunetti ND, Salvemini G, Cuculo A, et al. Coronary artery ectasia is related to coronary slow flow and inflammatory activation. Atherosclerosis 2014;233:636-40.

6 Demopoulos VP, Olympios CD, Fakiolas CN, et al. The natural history of aneurysmal coronary artery disease. Heart 1997;78:136-41.

7 Eitan A, Roguin A. Coronary artery ectasia. Coron Artery Dis 2016;27:420-8.

8 Hartnell GG, Parnell BM, Pridie RB. Coronary artery ectasia. its prevalence and clinical significance in 4993 patients. Br Heart $J$ 1985;54:392-5.
9 Lam CSP, Ho KT. Coronary artery ectasia: a ten-year experience in a tertiary hospital in Singapore. Ann Acad Med Singapore 2004;33:419-22.

10 Zografos TA, Korovesis S, Giazitzoglou E, et al. Clinical and angiographic characteristics of patients with coronary artery ectasia. Int J Cardiol 2013;167:1536-41.

11 Krüger D, Stierle U, Herrmann G, et al. Exercise-induced myocardial ischemia in isolated coronary artery ectasias and aneurysms ("dilated coronopathy"). J Am Coll Cardiol 1999;34:1461-70.

12 Akyürek O, Berkalp B, Sayin T, et al. Altered coronary flow properties in diffuse coronary artery ectasia. Am Heart $J$ 2003;145:66-72.

13 Mavrogeni SI, Manginas A, Papadakis E, et al. Coronary flow evaluation by TIMI frame count and magnetic resonance flow velocity in patients with coronary artery ectasia. J Cardiovasc Magn Reson 2005;7:545-50.

14 Ismail AM, Rayan M, Adel A, et al. Prevalence and pattern of abnormal myocardial perfusion in patients with isolated coronary artery ectasia: study by $99 \mathrm{mTc}$-sestamibi radionuclide scintigraphy. Int J Cardiovasc Imaging 2014;30:425-30.

15 Doi T, Kataoka Y, Noguchi T, et al. Coronary artery ectasia predicts future cardiac events in patients with acute myocardial infarction. Arterioscler Thromb Vasc Biol 2017;37:2350-5. 